\title{
In situ determination of induced dipole moments of pure and membrane-bound retinal chromophores
}

\author{
Th. Rasing* \\ Department of Physics, University of California, Berkeley, California 94720 \\ and Center for Advanced Materials, Lawrence Berkeley Laboratory, Berkeley, California 94720 \\ J. Huang and A. Lewis \\ Department of Applied Physics, Cornell University, Ithaca, New York 14853 \\ T. Stehlin and Y. R. Shen \\ Department of Physics, University of California, Berkeley, California 94720 \\ and Center for Advanced Materials, Lawrence Berkeley Laboratory, Berkeley, California 94720
}

(Received 22 August 1988; revised manuscript received 27 February 1989)

\begin{abstract}
By using the technique of optical second-harmonic generation, we have succeeded in obtaining the orientation, spectrum, and the change in dipole moment upon optical excitation for monolayers of retinal chromophores, which are of relevance for visual excitation. The change in dipole moment of the chromophore embedded in the purple membrane of Halobacterium halobium is found to be identical to that of the free chromophore. The spectrum of all-trans retinal monolayers exhibits a subsidiary one-photon forbidden peak in addition to the main absorption peak.
\end{abstract}

The molecular basis of visual excitation is a subject of considerable interest in modern biophysics. A visual pigment consists of a chromophore covalently bound to a protein molecule embedded in a membrane. Light energy absorbed in the chromophore is converted via a series of chemical reactions into a proton gradient across the membrane that eventually leads to a signal to the optical nerve. Bacteriorhodopsin (bR) is a protein related to visual pigments that is found in the purple membrane that grows in the bacterium Halobacterium halobium. ${ }^{1}$ In $\mathrm{bR}$ and all visual pigments the chromophore is a derivative of retinal (vitamin A aldehyde). There are many striking similarities between $b R$ and visual pigments, such as the spectral changes induced as a result of photon absorption. Because it is generally believed that one of the primary events in $\mathrm{bR}$ and the visual process is a photoisomerization of the retinylidene chromophore in the excited state, a detailed knowledge of the excited-state properties of the chromophore is essential. 2,3 Experimentally, this is hard to obtain, especially for the most relevant case of a chromophore inside the optical membrane. In particular, one has never been able to measure the change in dipole moment upon excitation of the chromophore in the membrane, a quantity important for modeling the photochemistry. ${ }^{4}$

In this paper, we show how one can use the technique of optical second-harmonic generation (SHG) to study monolayers of retinal spread at an air-water interface to obtain information about the orientation, excited states, and difference in dipole moment $\Delta \mu$ between the excited and ground state of the chromophore in its pure form and in the membrane-bound $b R$.

The effectiveness of SHG as a surface and monolayer probe stems from the fact that in the electric-dipole ap- proximation, SHG is forbidden in centrosymmetric media, but necessarily allowed at interfaces. ${ }^{5}$ Recently it has been shown that SHG can be used to obtain the hyperpolarizability of some organic molecules by spreading a monolayer of such molecules on water in a LangmuirBlodgett (LB) trough. ${ }^{6}$ This has the advantage of studying molecules in a more controlled way. Also, biological membranes can be simulated by LB films. In most cases the bulk contribution of the water substrate to the SHG signal can be neglected. In other cases it can be subtracted.

The SHG from a monolayer of molecular adsorbates is governed by a surface nonlinear polarization

$$
\mathbf{P}_{s}^{(2)}(2 \omega)=\overleftrightarrow{\chi}_{s}^{(2)}: \mathbf{E}(\omega) \mathbf{E}(\omega)
$$

With local-field corrections neglected, the nonlinear susceptibility $\overleftrightarrow{\chi}_{s}^{(2)}$ is related to the molecular hyperpolarizability $\overleftrightarrow{\alpha}^{(2)}$ by

$$
\chi_{s, i j k}^{(2)}=N_{s}\left\langle T_{i j k}^{\lambda \mu v}\right\rangle \alpha_{\lambda \mu \nu}^{(2)} .
$$

Here, $N_{s}$ is the surface density of the molecules and $\left\langle T_{i j k}^{\lambda \mu v}\right\rangle$ describes the coordinate transformation between the molecular $(\zeta, \eta, \epsilon)$ system and the laboratory $(x, y, z)$ system, averaged over the molecular orientations.

By a proper combination of input and output polarizations, various $\chi_{s, i j k}^{(2)}$ components are accessible. To obtain $\overleftrightarrow{\alpha}^{(2)}$, a detailed knowledge of $\left\langle T_{i j k}^{\lambda \mu v}\right\rangle$ is necessary, but generally this is not always possible. However, the situation is greatly simplified when $\overleftrightarrow{\alpha}^{(2)}$ is dominated by a single component along one of the molecular axis $\hat{\boldsymbol{\epsilon}}$ and the latter has a random azimuthal distribution around the surface normal $\widehat{\mathbf{z}}$. The nonvanishing elements of $\vec{\chi}_{s}^{(2)}$ can then be written as ${ }^{6}$ 
$\chi_{s, z z z}^{(2)}=N_{s}\left\langle\cos ^{3} \theta\right\rangle \alpha_{\epsilon \epsilon \epsilon}^{(2)}$

$\chi_{s, z i i}^{(2)}=\chi_{s, i z i}^{(2)}=\chi_{s, i i z}^{(2)}=\frac{1}{2} N_{s}\left\langle\sin ^{2} \theta \cos \theta\right\rangle \alpha_{\epsilon \epsilon \epsilon}^{(2)}$,

$i=x$ or $y$,

where $\theta$ is the polar angle between $\hat{\boldsymbol{\epsilon}}$ and $\hat{\mathbf{z}}$. For an ordered monolayer of retinal molecules on a water surface, the orientational distribution is expected to sharply peak in a certain direction and can be approximated by a $\delta$ function. Then, from Eq. (3) it follows that a measurement of any two linear combinations of $\chi_{s, z z z}^{(2)}$ and $\chi_{s, z i i}^{(2)}$ will yield a value for $\alpha_{\epsilon \epsilon \epsilon}^{(2)}$ and $\theta .^{6}$

The two lowest singlet excited states of retinal possess $B_{u}$-like and $A_{g}$-like symmetry, assuming an effective $C_{2 h}$ point-group symmetry for the molecule. Excitation into the first excited $\pi \pi^{*}\left(B_{u}\right)$ state is accompanied by a large charge transfer along the long axis of the molecule. ${ }^{7,8}$ In such a case $\alpha_{\epsilon \epsilon \epsilon}^{(2)}$ dominates and can be described by ${ }^{9}$

$$
\alpha_{\epsilon \epsilon \epsilon}^{(2)}=\alpha_{\mathrm{ct}}^{(2)}+\alpha_{\mathrm{nr}}^{(2)} .
$$

Here, $\alpha_{\mathrm{ct}}^{(2)}$ is the contribution due to the charge transfer connected with the main optical absorption band of the chromophore and $\alpha_{\mathrm{nr}}^{(2)}$ is the total nonresonant contribution. Assuming a two-level system, $\alpha_{\mathrm{ct}}^{(2)}$ can be written as $^{9,10}$

$$
\alpha_{\mathrm{ct}}^{(2)}=\frac{3 e^{2}}{2 m \hbar} \frac{\omega_{0}}{\left(\omega_{0}^{2}-\omega^{2}\right)\left(\omega_{0}^{2}-4 \omega^{2}\right)} f \Delta \mu,
$$

where $\hbar \omega_{0}$ is the excitation energy, $f$ its oscillator strength, $\Delta \mu$ the difference between the dipole moment of the excited state and the ground state, and $\hbar \omega$ is the energy of the incident laser.

The validity of Eq. (5) can be checked by comparing the $\alpha^{(2)}$ values as obtained from the SHG results with known values of $\Delta \mu$ for a series of retinal derivatives. Knowing that Eq. (5) holds, we can then use SHG as a probe to measure the unknown $\Delta \mu$ of the chromophore in the membrane. Equation (5) also shows that SHG can be exploited to obtain information about the excitation energy $\hbar \omega_{0}$ : by scanning the incident pump frequency $\omega, \alpha^{(2)}$ will be resonantly enhanced whenever $\omega_{0}=\omega$ or $\omega_{0}=2 \omega$.

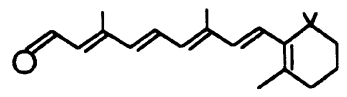

A T R

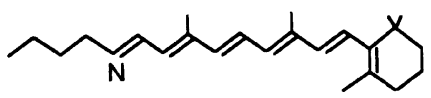

NRB

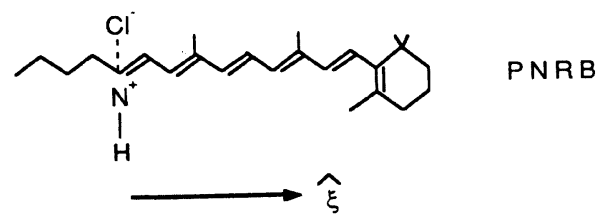

FIG. 1. Structure of molecules studied (see text for abbreviations).

[Note that close to resonance, Eq. (5) has to be modified to include damping. ${ }^{10}$ ]

For the SHG experiments we used a $Q$-switched $\mathrm{Nd}$ :YAG (YAG is yttrium aluminum garnet) laser at $1.064 \mu \mathrm{m}$ and its frequency-doubled output at $532 \mathrm{~nm}$ as the pump beam. For frequency tuning, a Nd:YAG pumped dye laser was used. The intensity at the surface was less than $20 \mathrm{~mW}$ focused onto a $7-\mathrm{mm}^{2}$ spot. The surface SHG signal was calibrated against the signal from a thin quartz crystal with a bulk nonlinear susceptibility $\chi_{s, x x x}^{(2)}=2.2 \times 10^{-9}$ esu. $^{11}$

To establish the relation between $\alpha^{(2)}$ and $\Delta \mu$ we measured $\alpha^{(2)}$ of three retinal derivatives: all-trans retinal (ATR), all-trans retinylidene butylamine Schiff base (NRB), and protonated NRB (PNRB), for all of which $\Delta \mu$ is known. ${ }^{8}$ Figure 1 shows the structure of the molecules and the choice of the molecular $\hat{\boldsymbol{\epsilon}}$ axis.

Monolayers were prepared by spreading an appropriate amount of solution of the molecules in methanol or hexane on the water surface. Because NRB and PNRB are slightly soluble in water, their SH intensities were compared with those obtained from spin coated monolayers on glass substrates, carefully prepared to get the same surface density of molecules. We found good agreement in the two cases.

Table I summarizes the measured values of $\alpha^{(2)}$ and the comparison with Eq. (5). The oscillator strengths in

TABLE I. Molecular hyperpolarizability $\alpha^{(2)}$ and change in dipole moment upon excitation $\Delta \mu$ for the retinal chromophores studied. $\lambda_{0}$ equals the peak position of main absorption band and $f$ equals the oscillator strength. The $\Delta \mu$ values are given relative to that of ATR at $1.064 \mu \mathrm{m}$.

\begin{tabular}{lccccccc}
\hline \hline & $\begin{array}{c}\lambda_{0} \\
(\mathrm{~nm})\end{array}$ & $f^{\mathrm{a}}$ & $\begin{array}{c}\alpha^{(2) \mathrm{b}} \\
\left(10^{-28} \mathrm{esu}\right)\end{array}$ & $\begin{array}{c}\alpha^{(2) \mathrm{c}} \\
\left(10^{-28} \mathrm{esu}\right)\end{array}$ & $\Delta \mu^{\mathrm{b}}$ & $\Delta \mu^{\mathrm{c}}$ & $\Delta \mu^{\mathrm{d}}$ \\
\hline ATR & 380 & 0.81 & 1.4 & 1.3 & 1.30 & 1 & 1 \\
NRB & 365 & 0.98 & 1.2 & 1.1 & 0.93 & 0.84 & 0.69 \\
PNRB & 430 & 0.91 & 2.3 & 2.5 & 1.50 & 0.86 & 1.03 \\
PNRB $^{*}$ & 566 & 0.90 & 1.5 & 11.0 & 0.60 & 0.91 & \\
\hline \hline
\end{tabular}

${ }^{a}$ From Ref. 12.

${ }^{\mathrm{b}}$ At $532 \mathrm{~nm}$.

${ }^{\mathrm{c}}$ At $1.064 \mu \mathrm{m}$.

${ }^{\mathrm{d}}$ From Ref. 10. 
Table I were obtained from the absorption spectra of the various molecules. ${ }^{12}$ The results show that the retinal molecules indeed have a very large nonlinearity $\left(\alpha^{(2)} \sim 10^{-28}\right.$ esu) that is dominated by the resonant part $\alpha_{\mathrm{ct}}^{(2)}$ : Within the experimental errors $(\sim 15 \%)$ the relative values of $\Delta \mu$ obtained from the SHG results using Eq. (5) correlate quite well with those obtained from the Stark-shift measurements. ${ }^{8}$ [Note: The absolute values of $\Delta \mu$ as calculated from Eq. (5) are a factor of 2 larger than those from Ref. 8, which is still a fairly good agreement considering all the local-field corrections that one has to take into account to analyze Stark-shift data and the fact that here all local-field effects have been neglected.]

In order to obtain further direct information about the excited states of the chromophore and the effect on $\Delta \mu$ in the monolayer structure, we have measured $\chi_{s}^{(2)}(\omega)$ for a monolayer of all-trans retinal by scanning the pump beam and measuring the corresponding SHG signal. The results plotted in Fig. 2 show two resonances: one at $\omega=335 \mathrm{~nm}$ and one at $\omega=360 \mathrm{~nm}$. The main absorption band of all-trans retinal in solution peaks at $380 \mathrm{~nm}$ (at $300 \mathrm{~K})$. Two-photon spectroscopy at $77 \mathrm{~K}$ shows the same band at $388 \mathrm{~nm}$ as well as an additional $A_{g}$-like band at $429 \mathrm{~nm} .^{13}$ Based on this information we can assign the two resonances as due to the $B_{u}$-like main absorption band at $335 \mathrm{~nm}$ and the one-photon-forbidden $A_{g}$-like band at $360 \mathrm{~nm}$. Both states appear to be blue shifted from those of molecules in solution by approximately the same amount.

Our observation of the one-photon-forbidden excited state is also very interesting. Such states are believed to be important for the biological function of many photonreceptor chromophores due to their proximity and mixing with the main one-photon-allowed absorption band, but so far as we know they have never been observed for a single chromophore monolayer. The effect of the

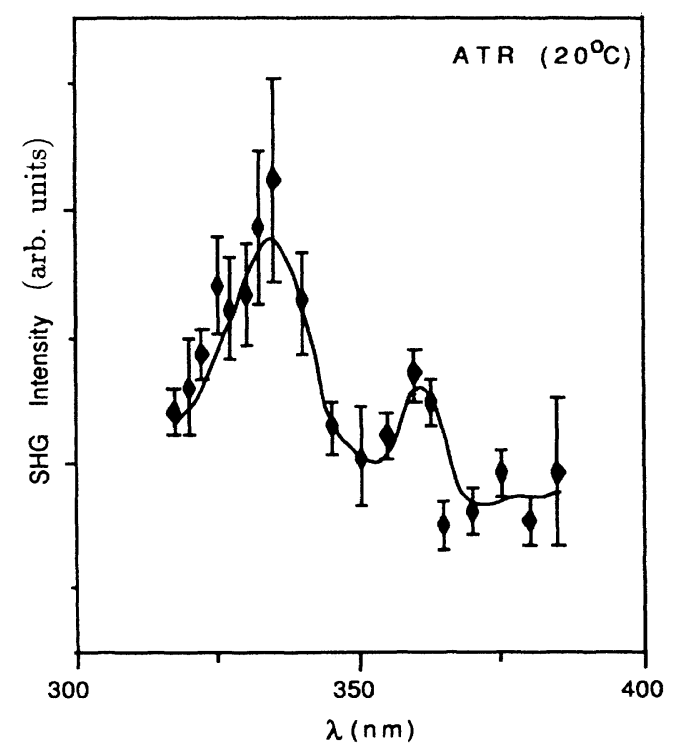

FIG. 2. SHG spectrum of all-trans retinal at an air-water interface. above-mentioned blue shift on the deduction of $\Delta \mu$ is negligible for all-trans retinal $(\sim 1 \%)$. The good agreement between $\Delta \mu$ derived from our experiment and those from Ref. 8 suggests that the similar spectral shifts of NRB and PNRB monolayers on water versus molecules in solution should not be very significant. These spectra have not yet been measured, but work is in progress to do so. (For multilayers of PNRB on glass, the linear absorption appeared to be blue shifted to $430 \mathrm{~nm}$ from $445 \mathrm{~nm}$ in solution.)

With this information about the excited states we can now exploit the correlation between $\alpha^{(2)}$ and $\Delta \mu$ by using SHG to determine $\Delta \mu$ of PNRB in a monolayer of purple membrane of Halobacterium halobium $\left[\Delta \mu\left(\mathrm{PNRB}^{*}\right)\right]$. In order to make a quantitative comparison, the effect of different dielectric environments between the free and bounded chromophores cannot be neglected. This can be estimated as follows. The boundary conditions for the electromagnetic fields at the interface lead to a factor of $1 / \epsilon_{z}^{\prime}$ in the Fresnel factors, where $\epsilon^{\prime}$ indicates the dielectric constant of the interface layer. ${ }^{14}$ As a result, what we have measured is the effective susceptibility $\chi_{s, \text { eff }}^{(2)}=\chi_{s}^{(2)} / \epsilon_{z}^{\prime}$. Also, due to the large red shift of the chromophore absorption band in the membrane (from 445 to $568 \mathrm{~nm}$ ), we have to modify Eq. (5) to include damping, ${ }^{10}$ because either the excitation wavelength (when we use $532 \mathrm{~nm}$ ) or the SHG wavelength (when we use $1.064 \mu \mathrm{m}$ as excitation) is close to the charge-transfer resonance.

Including all these effects one can write

$\frac{\Delta \mu\left(\text { PNRB }^{*}\right)}{\Delta \mu(\text { PNRB })}=\frac{\chi_{\mathrm{eff}}^{(2)}\left(\mathrm{PNRB}^{*}\right) \epsilon_{\mathrm{PNRB}}^{*} N_{s} g(\theta) C_{\omega}}{\chi_{\mathrm{eff}}^{(2)}(\mathrm{PNRB}) \epsilon_{\mathrm{PNRB}} N_{s}^{*} g^{*}(\theta) C_{\omega}^{*}}$.

Here, $g(\theta)=\frac{1}{2} \sin ^{2} \theta \cos \theta, C_{\omega}=\alpha^{(2)} / \Delta \mu$ (including the effects of oscillator strength and damping), and the superscript * denotes the membrane case. For the pure PNRB monolayer at $N_{\delta}=2.5 \times 10^{14} \mathrm{~cm}^{-2}$ and a linear polarizability $\alpha^{(1)}=80 \AA^{3}$ (see Ref. 8), it follows from Ref. 15 that $\epsilon_{\mathrm{PNRB}} \simeq 1$. In the membrane, the chromophore molecules are farther apart $\left(N_{s} / N_{s}^{*}=50\right)$, so that the local-field effects due to the intermolecular interactions on $\epsilon$ can be neglected and $\epsilon_{\text {PNRB }}^{*}$ mainly results from the surrounding protein molecules. Due to their large hydrocarbon content, we estimate that the index of refraction of the membrane is 1.5 , yielding $\epsilon_{\mathrm{PNRB}}^{*}=2.25$, which is similar to the choice of $\epsilon=2$ for the protein environment in Ref. 8. From $\theta^{*}=66^{\circ}$ (Ref. 16) and $\theta=50^{\circ}$ (from our SHG results), we find $g(\theta) / g^{*} /(\theta)=1.12$. With $C_{\omega} / C_{\omega}^{*}=0.6$, this yields $\Delta \mu\left(\right.$ PNRB $\left.^{*}\right) / \Delta \mu($ PNRB $)$ $=0.4$ for the $532-\mathrm{nm}$ excitation wavelength. The problem with this latter excitation is that it bleaches the membrane, ${ }^{12}$ which does not happen at $1.064 \mu \mathrm{m}$. There we find $\Delta \mu\left(\right.$ PNRB $\left.^{*}\right) / \Delta \mu($ PNRB $)=1.1$ (see also Ref. 12).

These results show that the change in dipole moment of the PNRB chromophore embedded in the membrane is about equal to that found for the free chromophores. To our knowledge, this is the first time that this important parameter has ever been determined experimentally. Of course, the result for $\Delta \mu$ depends on the choice of $\epsilon^{*}$. As an additional test, we have purposely bleached the mem- 
brane to modify the chromophores only. The results were consistent with those reported above and indicated that our choice for $\epsilon^{*}$ is reasonable.

In conclusion, we have shown how the monolayer sensitivity of optical second-harmonic generation can be exploited to obtain information about the biologically very important retinal chromophores. We have measured the change in dipole moment upon optical excitation in the chromophore inside an optical membrane for the first time and found it to be the same as for the free chromophore. We have also shown that SHG provides a fairly simple way to obtain spectral information about the chromophores, and measured both the $B_{u}$-like and $A_{g}$-like states of a monolayer of all-trans retinal at an air-water interface. Finally, we believe that this technique could readily be extended to other relevant biological and chemical applications which require the investigation of dipolar and structural relationships between related systems.

We thank H. Hsiung for useful discussions and a critical reading of the manuscript. T.S. acknowledges support from Deutsche Forschungsgemeinschaft, Federal Republic of Germany. Part of this work was supported by the Director, Office of Energy Research, Office of Basic Energy Sciences, Materials Sciences Division of the U.S. Department of Energy under Contract No. DEAC03-76SF00098, and part by the U.S. Army, Contract No. DAMD17-85C-5136 and a grant from the U.S. Air Force to A.L.
${ }^{*}$ Present address: Faculty of Science, University of Nijmegen, 6525 ED Nijmegen, The Netherlands.

${ }^{1}$ W. Stoeckenius and R. A. Bogomolni, Ann. Rev. Biochem. 52, 587 (1982).

${ }^{2}$ R. R. Birge, Ann. Rev. Biophys. Bioeng. 10, 315 (1981).

${ }^{3}$ M. Ottolenghi, Adv. Photochem. 12, 97 (1980).

${ }^{4}$ A. Lewis, Proc. Natl. Acad. Sci. U.S.A. 75, 549 (1978).

${ }^{5}$ Y. R. Shen, J. Vac. Sci. Technol. B 3, 1464 (1985).

${ }^{6}$ Th. Rasing, G. Berkovic, Y. R. Shen, S. G. Grubb, and M. W. Kim, Chem. Phys. Lett. 130, 1 (1986).

${ }^{7}$ R. R. Birge and L. M. Hubbard, J. Am. Chem. Soc. 102, 2195 (1980).

${ }^{8}$ M. Ponder and R. Mathies, J. Phys. Chem. 87, 5090 (1983).

${ }^{9}$ J. L. Oudar and D. S. Chemla, J. Chem. Phys. 66, 2664 (1977).

${ }^{10}$ N. Bloembergen and Y. R. Shen, Phys. Rev. A 37, 133 (1964).
${ }^{11}$ CRC Handbook of Lasers edited by R. J. Priestly (Chemical Rubber Co., Cleveland, 1971), p. 497.

12J. Huang, Z. Chen, and A. Lewis, J. Phys. Chem. 93, 3314 (1989).

${ }^{13}$ R. R. Birge, Acc. Chem. Res. 19, 138 (1986).

${ }^{14}$ T. H. Heinz, Ph.D. thesis, University of California at Berkeley, 1982; P. Guyot-Sionnest, W. Chen, and Y. R. Shen, Phys. Rev. B 33, 8254 (1986). The electromagnetic response of a monolayer can adequately be described by a macroscopic index of refraction [see Th. Rasing et al., Phys. Rev. A 37, 2732 (1988)] as has also been proven experimentally; see, e.g., T. Smith, J. Opt. Soc. Am. 58, 1069 (1968).

${ }^{15}$ P. Ye and Y. R. Shen, Phys. Rev. B 28, 4288 (1983).

${ }^{16}$ R. A. Bogomolni, S. B. Hwang, Y. W. Tseng, G. I. King, and W. Stoeckenius, Biophys. J. 17, 98a (1977). 